\title{
A Self-Adjusting Method to Resist Bus Bunching Based on Boarding Limits
}

\author{
Shuzhi Zhao, Chunxiu Lu, Shidong Liang, and Huasheng Liu \\ College of Transportation, Jilin University, Changchun 130022, China \\ Correspondence should be addressed to Shidong Liang; liangsd14@mails.jlu.edu.cn
}

Received 18 March 2016; Accepted 9 May 2016

Academic Editor: Hua Fan

Copyright ( 2016 Shuzhi Zhao et al. This is an open access article distributed under the Creative Commons Attribution License, which permits unrestricted use, distribution, and reproduction in any medium, provided the original work is properly cited.

\begin{abstract}
Bus bunching is one of the most serious problems of urban bus systems. Bus bunching increases waiting and travel time of passengers. Many bus systems use schedules to reach equal headways. Compared to the idea of schedules and the target headway introduced later, we propose a new method to improve the efficiency of a bus system and avoid bus bunching by boarding limits. Our solution can be effectively implemented when buses cannot travel as planned because of bad road conditions and dynamic demands at bus stops. Besides, using our method, bus headways reach the state with equal headways dynamically and spontaneously without drivers' explicit intervention. Moreover, the method can improve the level of the bus service and reduce total travel time of passengers. We verify our method using an ideal bus route and a real bus route, both showing the success of the proposed method.
\end{abstract}

\section{Bus Bunching}

It is always desirable for buses to run on a route with equal headways. Nevertheless, this is often difficult to accomplish due to complex conditions related to traffic on the road and demands from passengers at bus stops. Naturally, some buses become slow and some fast. Moreover, the phenomenon becomes more serious if no steps are taken. For example, if a bus is delayed for some reason, the bus will slow down and the headway between it and the bus before it will be larger than the normal headway. Therefore, more passengers will arrive at the next stop for the bus, which will cause even larger delay. Similarly, the headway between the bus and the bus behind it will become smaller than the expected headway, and there would be fewer passengers at the stop when the next bus arrives, which will make the next bus even faster. In other words, the fast bus will be faster, and the slow one will be slower. This phenomenon will exaggerate like a snowball, where small headways will become smaller and large headways will become larger, and if we do nothing, two buses will finally meet at some time, which is called bus bunching.

There are two main negative effects of bus bunching. One is that it increases the waiting time of passengers, because larger gaps allow more passengers to arrive at the stop and these passengers will wait for longer time. The other one is regarding wasting capacity, because fast buses take on fewer passengers and are nearly empty. So, in order to resist such negative effects, we provide an effective method.

We propose a self-adjusting method to equalize headways and provide superior quality service for passengers based on dynamic boarding limits. Our method does not use either the target headway or a schedule. We decide the number of passengers to be rejected to board on a control point according to both headways before and behind the bus. Headways will be adjusted spontaneously after a disruption. Drivers can drive carefully without having to check their time or locations. Passengers on a bus will not waste their time waiting for departure, and the management will be freed from designing a schedule.

\section{Overview of Solutions}

Bus bunching has long been understood and studied, yet it is still difficult to correct. Newell and Potts [1] propose the notion of bus bunching. To summarize previous studies, there are two groups of methods to resolve bus bunching. One is to make fast buses slow down, and the other is to make slow buses speed up, and then the headways between buses can be equalized. 
Barnett [2] focuses on computing the delays of buses after disruption. Turnquist and Blume [3] propose holding strategies to increase service regularity. Hickman [4] provides a comprehensive summary of previous studies on headway control. Fu and Yang [5] propose a scheme which can minimize the variation of headways and provide better service for passengers. Zhao et al. [6] propose a control model based on negotiation between two agents: one aboard the bus and the other at a stop. Zolfaghari et al. [7] discuss the objective that minimizes both passengers at a stop and those who have to wait for more than one bus. Puong and Wilson [8] extend their study by including extra waiting time endured by passengers into their objective function. Delgado et al. [9] develop a deterministic optimization model capable of incorporating holding and boarding limits. Cats et al. $[10,11]$ evaluate different holding strategies for improving service reliability. Ibarra-Rojas et al. [12] present a comprehensive review, discussing recent studies as well as works not addressed in previous reviews.

In the US, municipal bus routes are typically managed by target schedules. Under target schedules, the management has to spend a lot of time to make a reasonable schedule to provide the best service. And all the efforts will be useless if a bus lags behind the schedule.

Daganzo [13] proposes a scheme including the target headway to remit bus bunching. According to his model, there is a control point, and every bus arriving at the control point adjusts its headway according to the previous headway.

Bartholdi and Eisenstein [16] propose a self-coordinating measure. According to their model, the authors abandon the target schedule and target headway and delay a bus at the control point for a duration related to the headway so that headways tend to equalize even in the presence of perturbations.

Liang et al. [14] propose a self-adaptive control scheme to equalize the headways of buses with little slack in a single line automatically. In their method, they just need the information from the current bus at the control point, its leading bus, and following bus. Also they make contrast between the proposed method and previous self-equalizing methods to get conclusion.

Sánchez-Martínez et al. [15] formulate a mathematical model for holding control optimization that reflects dynamic running times and demand. Their model can be used to produce a plan of holding times for both the current state of the system and expected changes in running times and demand. The effectiveness of the model within a simulation environment is evaluated to show the advantages of their method.

Based on these studies, we provide our view on the methods that have been previously proposed or implemented. The traffic state and travel speeds of passengers are significantly influenced if we allow the fast bus to decelerate on an urban road. Besides, if we let the fast bus stop at a station for some extra time beyond what is necessary to take on the passengers, there will be some negative influence on the buses of other lines which stop at this station as well. Moreover, the passengers on board want to arrive at the destination quickly. Further, it is impossible to make a slow bus fast when the bus is running on an urban road because of the traffic. So the proposed method uses boarding limits at control stations to control buses.

We propose a self-adjusting control system based on the method of boarding limits, where we abandon the ideas of the target scheme, target headway, and bus holding. We successfully avoid unnecessary wasting of time at stations, and in our model buses stop at a station just to take on passengers. If the bus system is disturbed, the entire system will stabilize spontaneously in a short time without wasting time of passengers on board, where the headways on the route will spontaneously become equal.

\section{Self-Adjusting Method Based on Boarding Limits}

Consider an ideal system, a circle route with a control point, and $n$ buses running on it, where the average velocity of every bus is identical. The length of the route is $L$, and the direction of buses running on it is from 0 to $L$. $L$ is, in fact, the same point of the circle as 0 . Rejection will be implemented at the control point. Let the bus that just arrived at the control point be bus 1 , let the one before bus 1 in the direction of travel be bus 2 , and let the bus behind bus 1 be bus $n$. The headway $h_{i}$ is the time in which a bus covers the distance between bus $i$ and bus $i+1$. Also the headway $h_{n}$ is the time bus $n$ needs to run from its current location to the location of bus 1 . For each moment $t$, we use the vector $\mathbf{l}^{\mathbf{t}}=\left(l_{1}^{t}, l_{2}^{t}, \ldots, l_{n}^{t}\right)$ to express the locations of the buses at time $t$, where $0=l_{1}^{t} \leq l_{2}^{t} \leq \cdots \leq l_{n}^{t} \leq$ $L$. Similarly, we use the vector $\mathbf{h}^{\mathbf{t}}=\left(h_{1}^{t}, h_{2}^{t}, \ldots, h_{n}^{t}\right)$ to express the headways of the buses at time $t$, where $h_{i}^{t}=\left(l_{i+1}^{t}-l_{i}^{t}\right) / v$ for all buses $(2 \leq i \leq n-1)$ and $h_{n}^{t}=\left(L-l_{n}^{t}\right) / v$ for bus $n$, except for bus 1 , which will stop at the control point so that its headway will be changed to equalize the headways.

3.1. Ideal Model Using Dynamic Boarding Limits. When a bus (bus 1) arrives at the control point, we consider the relation between $h_{1}^{t}$ and $h_{n}^{t}$ and then decide if the rejection is required. We consider just the two adjacent headways $\left(h_{1}^{t}\right.$ and $h_{n}^{t}$, $t=1,2, \ldots, m)$ and our goal is to make the two adjacent headways equal after the three buses related to $h_{1}^{t}$ and $h_{n}^{t}$ pass through the control point. A sketch map is presented in Figure 1. Some parameters used in our formulas and their implications are described as follows:

$R^{t}$ : the number of passengers who arrive at the control point between time $t$ and time $t+1$.

$x^{t}$ : the number of the passengers that should be refused by bus 1 at the control point at time $t$.

$p$ : the average time a passenger needs to get on the bus.

$w$ : the number of the passengers arriving at the control point in one minute.

$v$ : the average travel speed between two adjacent control points.

$t^{*}$ : the dwell time of bus 1 at the control point. 


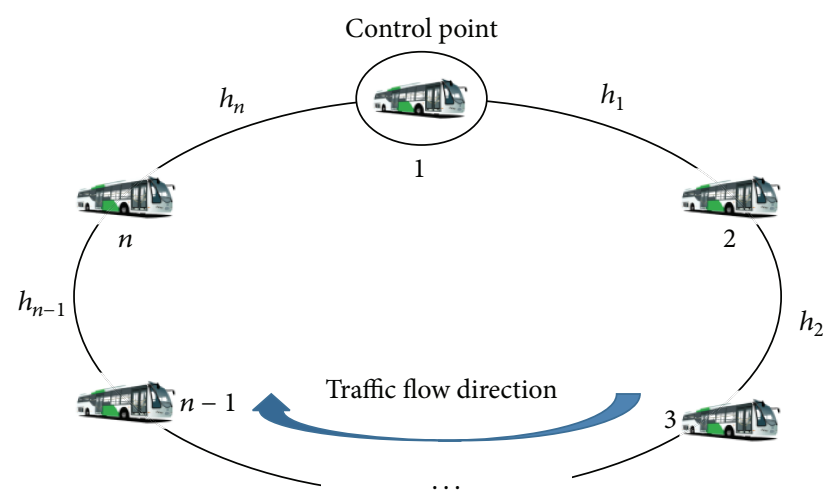

FIgURE 1: The bus system.

Theorem 1. When a bus arrives at the control point at time $t$, there are two situations: one is $x^{t} \leq 0$, and then we need not control the bus; the other is $x^{t}>0$, and then we will take rejection control. Considering the situation of $x^{t}>0$, if more than $x^{t}$ persons are waiting for a bus, $x^{t}$ persons should be rejected, and the other persons $\left(R^{t}-x^{t}\right)$ will get on; ifless than $x^{t}$ or just $x^{t}$ persons are waiting for a bus, all persons $\left(R^{t}\right)$ should be rejected, where

$$
x^{t}=\frac{h_{1}^{t}-h_{n}^{t}}{3 p}+\frac{2 \times R^{t}}{3}-\frac{h_{n}^{t} \times w}{3} .
$$

The headways of buses will stabilize at $H$ described as follows:

$$
H=\frac{L / v+n \times t^{*}}{n}=\frac{L}{n \times v(1-w \times p)} .
$$

Proof.

Time $t$. The location of the bus at the control point is 0 , which can be described as follows:

$$
l_{1}^{t}=0
$$

and the positions of buses change from moment $t$ to $t+1$ as described below.

Time $t+1$. First,

$$
l_{1}^{t+1}=0 .
$$

Second, the location of bus 2 at time $t+1$ can be calculated according to the location of bus 1 and the dwell time of bus 1 at the control point at time $t$, which can be described as $t^{*}=$ $\left(R^{t}-x^{t}\right) \times p .\left(R^{t}-x^{t}\right)$ means the number of the persons who will be taken, so the dwell time $t^{*}$ is decided by the number of the persons who will be taken $\left(R^{t}-x^{t}\right)$ and the average time a passenger needs to get on the bus $(p)$.

Therefore,

$$
\begin{aligned}
l_{2}^{t+1} & =l_{1}^{t}+\left(h_{n}^{t}-\left(R^{t}-x^{t}\right) \times p\right) \times v \\
& =\left(h_{n}^{t}-\left(R^{t}-x^{t}\right) \times p\right) \times v,
\end{aligned}
$$

and then we can obtain all other locations at time $t+1$ from the locations at time $t$ :

$$
l_{i}^{t+1}=l_{i-1}^{t}+h_{n}^{t} \times v \quad(i=3,4,5, \ldots, n) .
$$

Time $t+2$. Similarly, the positions of buses change from moment $t+1$ to $t+2$ as described below:

$$
\begin{aligned}
l_{1}^{t+2} & =0 \\
l_{2}^{t+2} & =l_{1}^{t+1}+\left(h_{n}^{t+1}-\left(R^{t+1}+x^{t}\right) \times p\right) \times v \\
& =\left(h_{n}^{t+1}-\left(R^{t+1}+x^{t}\right) \times p\right) \times v, \\
l_{i}^{t+2} & =l_{i-1}^{t+1}+h_{n}^{t+1} \times v \quad(i=3,4,5, \ldots, n) .
\end{aligned}
$$

As the time changes, the headways of buses at time $t+1$ change as follows. We use formula (5) to obtain

$$
h_{1}^{t+1}=\frac{l_{2}^{t+1}-l_{1}^{t+1}}{v}=\frac{l_{2}^{t+1}}{v}=h_{n}^{t}-\left(R^{t}-x^{t}\right) \times p .
$$

By combining formulas (5) and (6), we further have

$$
\begin{aligned}
h_{2}^{t+1} & =\frac{l_{3}^{t+1}-l_{2}^{t+1}}{v} \\
& =\frac{\left(l_{2}^{t}+h_{n}^{t} \times v\right)-\left(h_{n}^{t}-\left(R^{t}-x^{t}\right) \times p\right) \times v}{v} \\
& =\frac{l_{2}^{t}+\left(R^{t}-x^{t}\right) \times p \times v}{v}=\frac{l_{2}^{t}}{v}+\left(R^{t}-x^{t}\right) \times p \\
& =h_{1}^{t}+\left(R^{t}-x^{t}\right) \times p .
\end{aligned}
$$

Calculations for the other headways (formulas (12)) based on formula (6) and the relation between headways and locations are presented below:

$$
\begin{aligned}
h_{i}^{t+1} & =\frac{l_{i+1}^{t+1}-l_{i}^{t+1}}{v}=\frac{\left(l_{i}^{t}+h_{n}^{t} \times v\right)-\left(l_{i-1}^{t}+h_{n}^{t} \times v\right)}{v} \\
& =\frac{l_{i}^{t}-l_{i-1}^{t}}{v}=h_{i-1}^{t} \quad(i=3,4, \ldots, n-1), \\
h_{n}^{t+1} & =\frac{L-l_{n}^{t+1}}{v}=\frac{L-\left(l_{n-1}^{t}+h_{n}^{t} \times v\right)}{v} \\
& =\frac{L-\left(l_{n-1}^{t}+\left(\left(L-l_{n}^{t}\right) / v\right) \times v\right)}{v}=\frac{l_{n}^{t}-l_{n-1}^{t}}{v} \\
& =h_{n-1}^{t} .
\end{aligned}
$$

The headways of buses at time $t+2$ can be described as follows. The headway of bus 1 at time $t+2$ can be described by formula (13) according to (8), and the headway of bus 2 at 
time $t+2$ can be described by formula (14) according to (8), (9), and (10):

$$
\begin{aligned}
h_{1}^{t+2} & =\frac{l_{2}^{t+2}-l_{1}^{t+2}}{v}=\frac{l_{2}^{t+2}}{v} \\
& =\frac{\left(h_{n}^{t+1}-\left(R^{t+1}+x^{t}\right) \times p\right) \times v}{v} \\
& =h_{n}^{t+1}-\left(R^{t+1}+x^{t}\right) \times p, \\
h_{2}^{t+2} & =\frac{l_{3}^{t+2}-l_{2}^{t+2}}{v} \\
& =\frac{\left(l_{2}^{t+1}+h_{n}^{t+1} \times v\right)-\left(h_{n}^{t+1}-\left(R^{t+1}+x^{t}\right) \times p\right) \times v}{v} \\
& =\frac{l_{2}^{t+1}+\left(R^{t+1}+x^{t}\right) \times p \times v}{v} \\
& =\frac{l_{2}^{t+1}}{v}+\left(R^{t+1}+x^{t}\right) \times p=h_{1}^{t+1}+\left(R^{t+1}+x^{t}\right) \times p .
\end{aligned}
$$

Formulas (15) can be deduced from formula (9) as follows:

$$
\begin{aligned}
h_{i}^{t+2} & =\frac{l_{i+1}^{t+2}-l_{i}^{t+2}}{v} \\
& =\frac{\left(l_{i}^{t+1}+h_{n}^{t+1} \times v\right)-\left(l_{i-1}^{t+1}+h_{n}^{t+1} \times v\right)}{v} \\
& =\frac{l_{i}^{t+1}-l_{i-1}^{t+1}}{v}=h_{i-1}^{t+1} \quad(i=3,4,5, \ldots, n-1), \\
h_{n}^{t+2} & =\frac{L-l_{n}^{t+2}}{v}=\frac{L-\left(l_{n-1}^{t+1}+h_{n}^{t+1} \times v\right)}{v} \\
& =\frac{L-\left(l_{n-1}^{t+1}+\left(\left(L-l_{n}^{t+1}\right) / v\right) \times v\right)}{v}=\frac{l_{n}^{t+1}-l_{n-1}^{t+1}}{v} \\
& =h_{n-1}^{t+1} .
\end{aligned}
$$

Using the above, we can obtain the headway of every bus in the system at time $t+1$ and time $t+2$ :

$$
\begin{aligned}
& h_{1}^{t+1}=h_{n}^{t}-\left(R^{t}-x^{t}\right) \times p, \\
& h_{2}^{t+1}=h_{1}^{t}+\left(R^{t}-x^{t}\right) \times p, \\
& h_{i}^{t+1}=h_{i-1}^{t} \quad(i=3,4,5, \ldots, n), \\
& h_{1}^{t+2}=h_{n}^{t+1}-\left(R^{t+1}+x^{t}\right) \times p, \\
& h_{2}^{t+2}=h_{1}^{t+1}+\left(R^{t+1}+x^{t}\right) \times p, \\
& h_{i}^{t+2}=h_{i-1}^{t+1} \quad(i=3,4,5, \ldots, n) .
\end{aligned}
$$

In order to make the headways of two subsequent buses equal after the three buses related run through the control point, we use the following equation to calculate the number of passengers to be rejected:

$$
h_{3}^{t+2}=h_{2}^{t+2} .
$$

By substituting formula (21) for $i=3$ into formula (22), we can get the following relation:

$$
h_{2}^{t+1}=h_{2}^{t+2} .
$$

Combining formulas (17) and (20) with (23), the equation can be described as follows:

$$
h_{1}^{t}+\left(R^{t}-x^{t}\right) \times p=h_{1}^{t+1}+\left(R^{t+1}+x^{t}\right) \times p .
$$

We assume the relationship between the number of passengers arriving at the station and the headways of two buses as follows:

$$
\begin{aligned}
& R^{t}=h_{1}^{t} \times w \quad(t=1), \\
& R^{t}=h_{n}^{t-1} \times w \quad(t=2,3,4, \ldots, m) .
\end{aligned}
$$

Combining formulas (26) and (24), we get the following formula:

$$
h_{1}^{t}+\left(R^{t}-x^{t}\right) \times p=h_{1}^{t+1}+\left(h_{n}^{t} \times w+x^{t}\right) \times p .
$$

We further obtain formulas (28) as follows:

$$
\begin{aligned}
h_{1}^{t} & +2 \times p \times R^{t}-h_{n}^{t}-h_{n}^{t} \times w \times p=3 \times p \times x^{t}, \\
x^{t} & =\frac{h_{1}^{t}+2 \times p \times R^{t}-h_{n}^{t}-h_{n}^{t} \times w \times p}{3 \times p} \\
& =\frac{h_{1}^{t}-h_{n}^{t}}{3 \times p}+\frac{2 \times R^{t}}{3}-\frac{h_{n}^{t} \times w}{3} .
\end{aligned}
$$

So when a bus arrives at the control point at time $t$, if $x^{t} \leq$ 0 , no one will be rejected; if more than $x^{t}$ persons are waiting for a bus, $x^{t}$ persons should be rejected; and if less than $x^{t}$ or just $x^{t}$ persons are waiting for a bus, all persons $\left(R^{t}\right)$ should be rejected.

According to our model, after a few circles, the headway will be stabilized at a fixed value, derived as follows.

The stable headway is the average travel time of $n$ buses (29). Consider the derivation process in one circle, from the time a bus arrives at the control point to the time it arrives at the control point next time; each bus on the route runs one circle. And each bus passes the control point and drives passengers one time. So the total travel time of $n$ buses is composed of running time $(L / v)$ and total dwell time $\left(n \times t^{*}\right)$, and the headway between every two buses is the average of total time just as formula (29).

The dwell time of every bus at the control point is related to the number of passengers who are waiting for buses and the average time $(p)$ a passenger needs to get on the bus. In a stable system, no passengers should be resisted and headways are equal, so the number of passengers who are waiting for buses can be described as the number $\left(R^{t}=H \times w\right)$ of 
passengers who arrive at the control point between time $t$ and time $t+1$. Then we can obtain formula (30):

$$
\begin{aligned}
H & =\frac{\left(L / v+n \times t^{*}\right)}{n}, \\
H \times w \times p & =t^{*},
\end{aligned}
$$

where $L$ is the length of the route and $H$ is the stable headway. From formula (29) and formula (30), we can obtain the stable $t^{*}$ and $H$ as follows:

$$
\begin{aligned}
& t^{*}=\frac{L \times w \times p}{(1-w \times p) \times n \times v}, \\
& H=\frac{L / v+n \times t^{*}}{n}=\frac{L}{n \times v \times(1-w \times p)} .
\end{aligned}
$$

3.2. Verification of the Ideal Model. According to the formulas in Section 3.1, we assign a few values to the related parameters; namely, $n=4, h(1,1)=12(\min ), h(1,2)=9(\min )$, $h(1,3)=11(\mathrm{~min}), h(1,4)=8(\mathrm{~min}), p=0.05(\mathrm{~min})$, and $w=0.5$ (persons $/ \mathrm{min}$ ). The variation in headways can be described by the number of buses arriving at the control point; see Figure 2.

As can be seen in Figure 2, initially there are some differences in the four headways, but they disappear as buses pass the control point continually. In a short time, they converge to the same value. Within a certain error range (0.05), the headways stabilize after 28 buses pass the control point. Hence, in an ideal bus system, after a disturbance, our method can stabilize the system after a few circles, as shown in the numerical simulation.

\section{Verification on a Real Bus Route}

In Section 3, we have shown that our method can balance the headways under ideal conditions without considering passengers' arrivals and variations in speed. However, on a real bus route, passengers' arrival rate and speeds are the key factors affecting the operation of vehicles. So we consider the two factors in our verification and compare the method based on bus holding and our method based on rejection.

For our verification, we chose a circle bus route whose length is 20000 meters. The time range is from 6:00 to 20:00. There are 20 stations on the route. We set 3 control points; their locations are at 4000 meters, 10000 meters, and 16000 meters, respectively. There are 9 buses running on the route. We believe that the speed of buses varies with time. We assume that passengers arrive at stations according to the Poisson distribution.

Based on the assumptions, we compare the selfcoordinating bus route proposed by Bartholdi and Eisenstein [16] and our self-adjusting method based on boarding limits. In what follows, we call the self-coordinating bus route method 1 and the self-adjusting method based on boarding limits method 2. The headways in different states are shown in Figure 3.

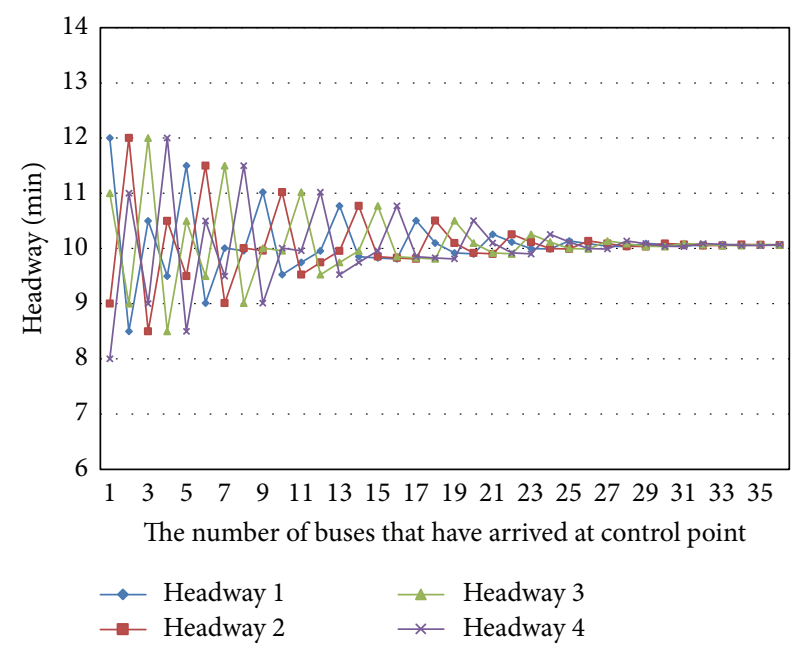

Figure 2: Variation in headways.

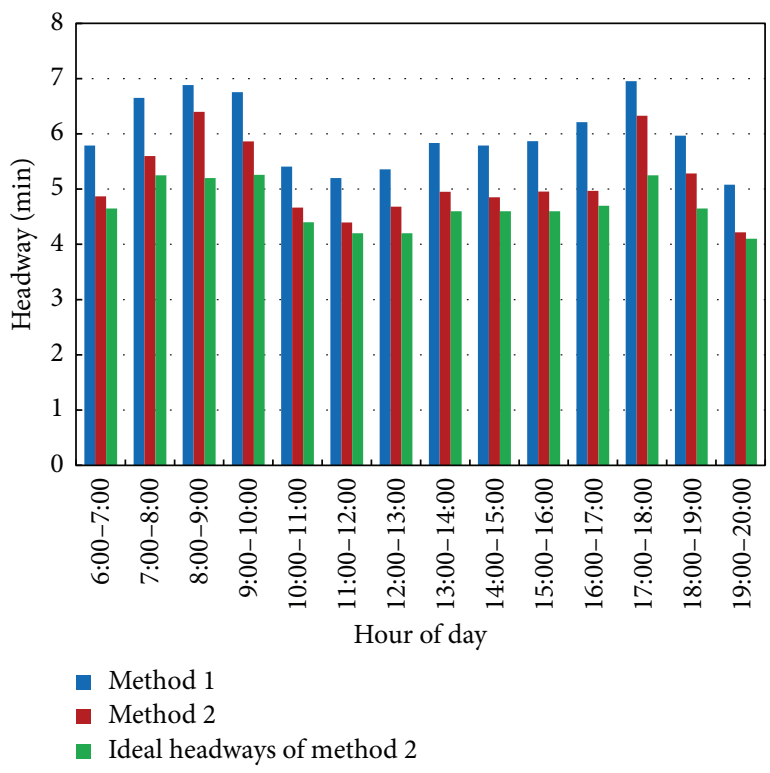

Figure 3: Comparison of headways.

In Figure 3, we provide the resulting headways of method 1 and method 2 based on the real bus route and method 2 based on the ideal bus route. Clearly, the headways of the ideal bus route are smaller than those of method 1 and method 2, and also the headways of method 2 (self-adjusting method based on rejection) are smaller than the headways of method 1 (self-coordinating method). The average headways of method 1 and method 2 are 5.98 minutes and 5.15 minutes, respectively. In other words, we can achieve better headways using our method based on rejection and cut down the spacing interval times between subsequent buses. As we can see in Figure 3, the headways are all smaller than 6 minutes except for the periods 8:00-9:00 and 17:00-18:00. However, there are five time periods in which headways of method 1 are larger than 6 minutes. Therefore, using our method, the 


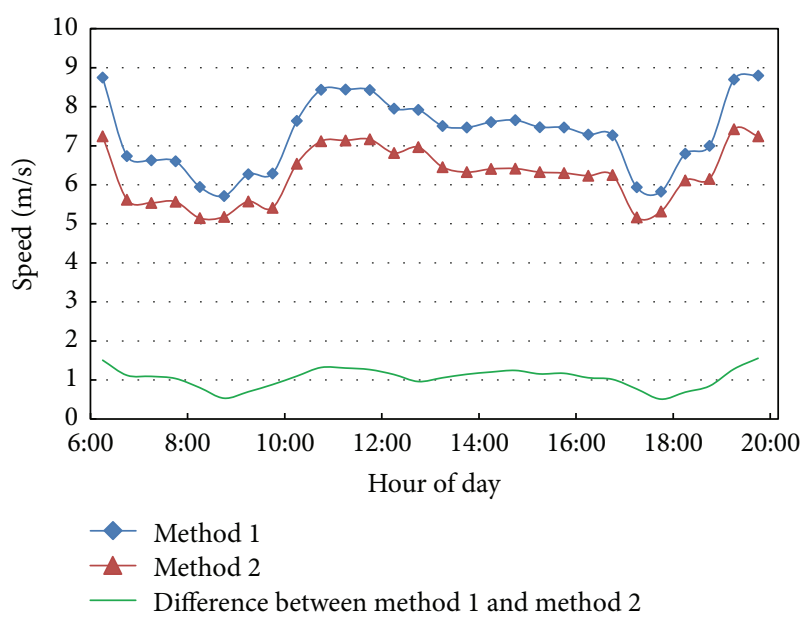

Figure 4: Travel speeds.

bus system can make one and a half circles more than using method 1.

Apart from headways of buses, the travel speed is another important factor of the performance of the bus system. In Figure 4, the travel speeds of method 1 and method 2 are shown. As can be seen in Figure 4, there is clear difference between the travel speeds of the two methods. Using our method, the travel speeds are larger than those of method 1 in every hour of the day. The average difference is about $1 \mathrm{~m} / \mathrm{s}$, and the average speed of method 2 increases by $16.8 \%$. The travel speeds fluctuate as the time changes in a day. During the morning peak and evening peak, our method 2 allows travel speed of about $6 \mathrm{~m} / \mathrm{s}$, but travel speed of method 1 is about $5 \mathrm{~m} / \mathrm{s}$. Hence, our method can maintain high travel speeds even during the morning or evening peak.

According to Figures 3 and 4, our method allows better headways and better travel speed. We also calculate the standard deviation of headways in Figure 5 to reflect the volatility. Clearly, the standard deviation of headways using our method is smaller than that of method 1 during all times. Even in the evening peak, our method can adjust headways with a small standard deviation.

Based on the comparison between method 2 and method 1 , headways, travel speeds, and standard deviations of headways using our method 2 are all better than those of method 1. And we compare the average travel times of passengers; see Table 1.

Comparing the results, although the average waiting time of passengers increases in our method, the time increases by just 0.69 minutes. At the same time, the average time of passengers on board decreases by 3.2 minutes. So the average travel time of passengers decreases by 2.51 minutes or by $10.7 \%$. So, overall, the total travel time of passengers is shorter in our method.

\section{Conclusion}

We first summarize previous studies and analyze their advantages and disadvantages. We chose the self-coordinating bus
TABLE 1: Waiting time and other time characteristics.

\begin{tabular}{lccc}
\hline Index & $\begin{array}{c}\text { Average } \\
\text { waiting time }\end{array}$ & $\begin{array}{c}\text { Average time } \\
\text { on board }\end{array}$ & $\begin{array}{c}\text { Average } \\
\text { travel time }\end{array}$ \\
\hline Method 1 (min) & 2.72 & 22.21 & 24.93 \\
Method 2 (min) & 3.41 & 19.01 & 22.42 \\
Value added (min) & 0.69 & -3.2 & -2.51 \\
Percent increase (\%) & 25.37 & -14.41 & -10.07 \\
\hline
\end{tabular}

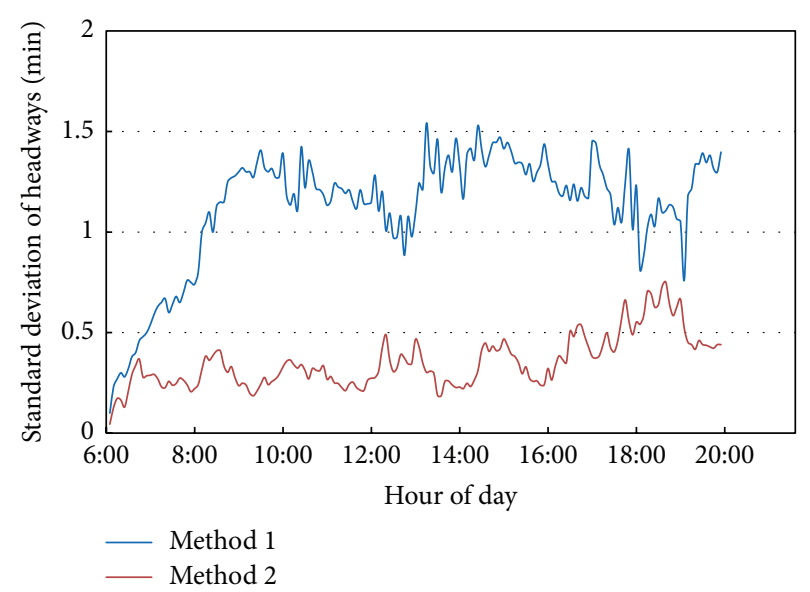

FIGURE 5: Standard deviations of headways.

route method that resists bus bunching to make comparison with our method and call it method 1 . The method provides satisfactory results. But the average travel time based on method 1 is too long, as bus holding affects the buses of other routes which stop at the same station.

We propose a self-adjusting method based on rejection. In our method, we abandon the idea of the target headway and schedule. According to our method, buses run all the time without wasting time except for stopping to take passengers. Also, drivers do not have to pay attention to time or schedule.

In comparison with other methods, we analyze headways, travel speeds, deviations of headways, and average travel times of passengers. All of these indexes reflect the advantages of our method based on boarding limits. Shorter headways, higher travel speeds, smaller deviations, and shorter travel times all certify that our method can resist bus bunching effectively and naturally and improve the quality of service.

\section{Competing Interests}

The authors declare that they have no competing interests.

\section{Acknowledgments}

The authors thank Bus Company of Changchun Public Traffic Group for valuable data. The work was supported by the National Natural Science Foundation of China (Grant no. 51378237). 


\section{References}

[1] G. Newell and R. Potts, "Maintaining a bus schedule," in Proceedings of the 2nd Australian Road Research Board (ARRB) Conference, pp. 388-393, Melbourne, Australia, 1964.

[2] A. Barnett, "On controlling randomness in transit operations," Transportation Science, vol. 8, no. 2, pp. 102-116, 1974.

[3] M. A. Turnquist and S. W. Blume, "Evaluating potential effectiveness of headway control strategies for transit systems," Transportation Research Record, no. 746, pp. 25-29, 1980.

[4] M. D. Hickman, "An analytic stochastic model for the transit vehicle holding problem," Transportation Science, vol. 35, no. 3, pp. 215-237, 2001.

[5] L. Fu and X. Yang, "Design and implementation of bus-holding control strategies with real-time information," Transportation Research Record, vol. 1791, pp. 6-12, 2002.

[6] J. Zhao, S. Bukkapatnam, and M. M. Dossouky, "Distributed architecture for real-time coordination of bus holding in transit networks," IEEE Transactions on Intelligent Transportation Systems, vol. 4, no. 1, pp. 43-51, 2003.

[7] S. Zolfaghari, N. Azizi, and M. Y. Jaber, "A model for holding strategy in public transit systems with real-time information," International Journal of Transport Management, vol. 2, no. 2, pp. 99-110, 2004.

[8] A. Puong and N. H. M. Wilson, "A train holding model for urban rail transit systems," in Computer-Aided Systems in Public Transport, pp. 319-337, Springer, Berlin, Germany, 2008.

[9] F. Delgado, J. C. Muñoz, R. Giesen, and A. Cipriano, "Real-time control of buses in a transit corridor based on vehicle holding and boarding limits," Transportation Research Record, vol. 2090, pp. 59-67, 2009.

[10] O. Cats, A. Larijani, H. N. Koutsopoulos, and W. Burghout, "Impacts of holding control strategies on transit performance: bus simulation model analysis," Transportation Research Record, vol. 2216, pp. 51-58, 2011.

[11] O. Cats, A. N. Larijani, Á. Ólafsdóttir, W. Burghout, I. J. Andréasson, and H. N. Koutsopoulos, "Bus-holding control strategies: simulation-based evaluation and guidelines for implementation," Transportation Research Record, no. 2274, pp. 100-108, 2012.

[12] O. J. Ibarra-Rojas, F. Delgado, R. Giesen, and J. C. Muñoz, "Planning, operation, and control of bus transport systems: a literature review," Transportation Research Part B: Methodological, vol. 77, pp. 38-75, 2015.

[13] C. F. Daganzo, "A headway-based approach to eliminate bus bunching: systematic analysis and comparisons," Transportation Research Part B: Methodological, vol. 43, no. 10, pp. 913-921, 2009.

[14] S. Liang, S. Zhao, C. Lu, and M. Ma, "A self-adaptive method to equalize headways: numerical analysis and comparison," Transportation Research B: Methodological, vol. 87, pp. 33-43, 2016.

[15] G. Sánchez-Martínez, H. N. Koutsopoulos, and N. H. M. Wilson, "Real-time holding control for high-frequency transit with dynamics," Transportation Research Part B: Methodological, vol. 83, pp. 1-19, 2016.

[16] J. J. Bartholdi and D. D. Eisenstein, "A self-coördinating bus route to resist bus bunching," Transportation Research Part B: Methodological, vol. 46, no. 4, pp. 481-491, 2012. 


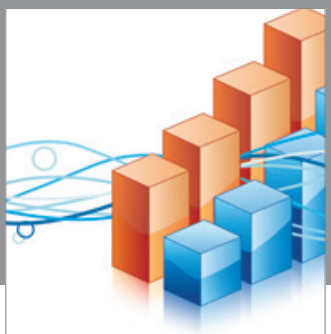

Advances in

Operations Research

vatem alat4

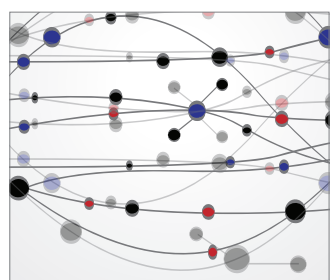

\section{The Scientific} World Journal
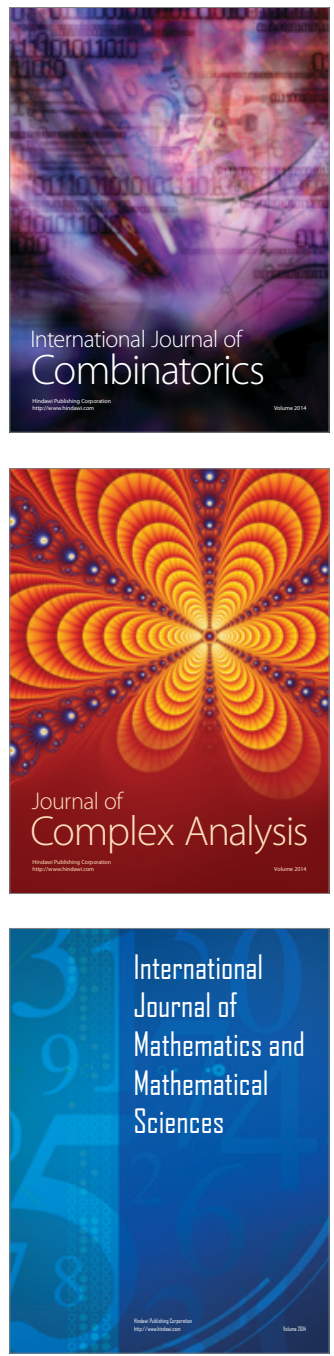
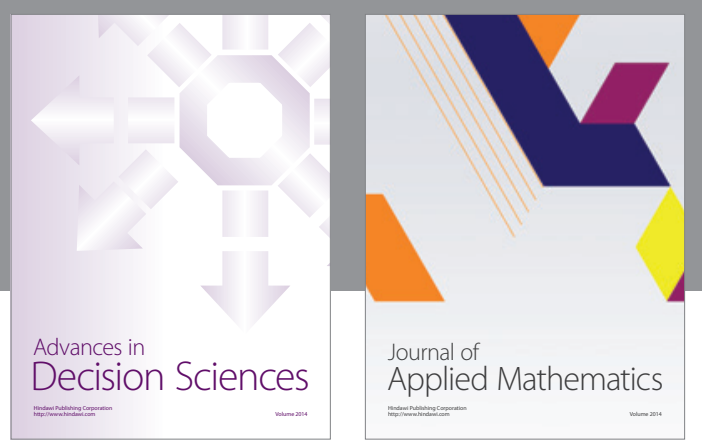

Algebra

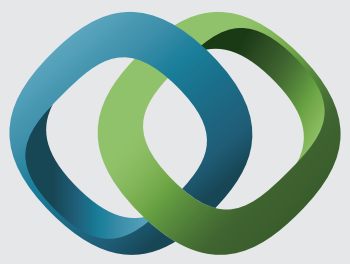

\section{Hindawi}

Submit your manuscripts at

http://www.hindawi.com
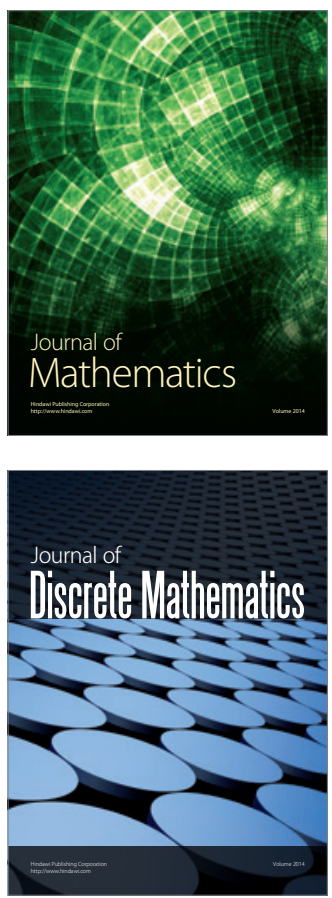

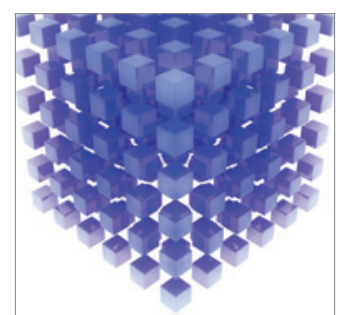

Mathematical Problems in Engineering
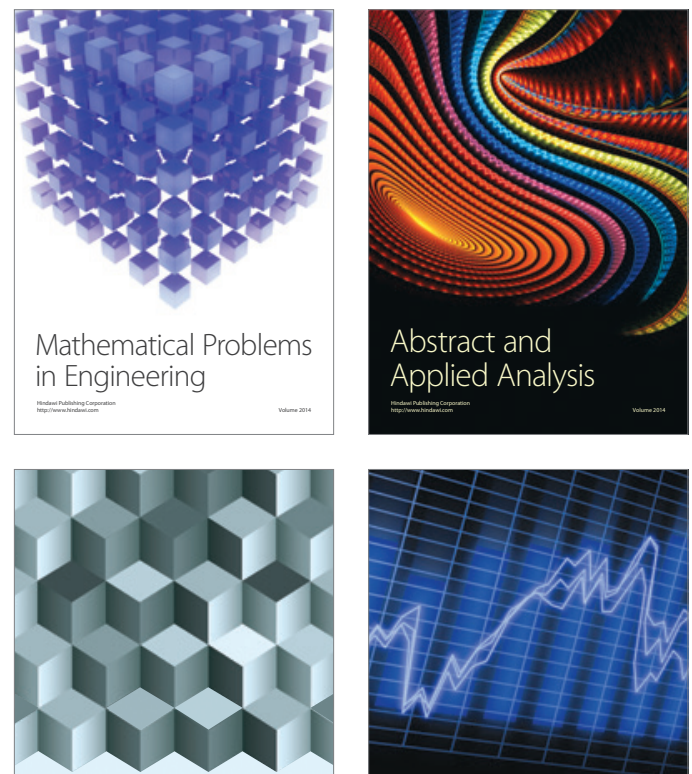

Journal of

Function Spaces

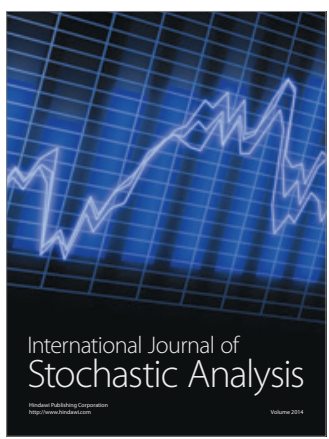

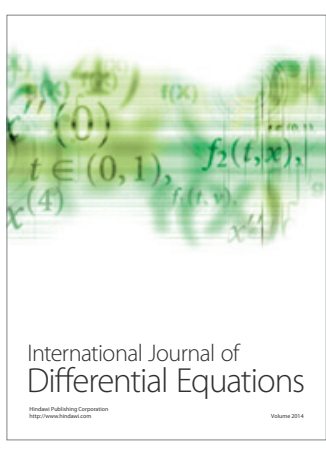
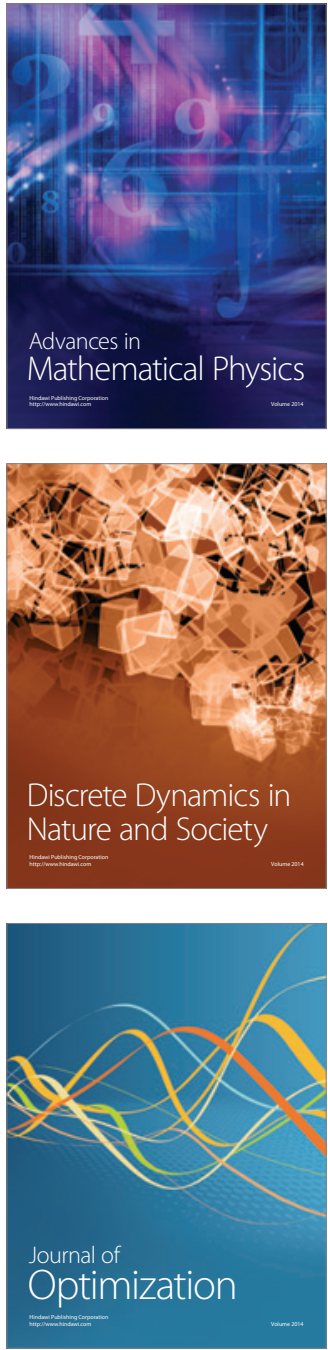